\title{
Enhanced diffusion of a tracer particle in a lattice model of a crowded active system
}

\author{
Leila Abbaspour $\odot^{*}$ and Stefan Klumpp $\oplus^{\dagger}$ \\ Institute for the Dynamics of Complex Systems and Max Planck School Matter to Life, University of Göttingen, \\ Friedrich-Hund-Platz 1, 37077 Göttingen, Germany
}

(Received 14 October 2020; accepted 15 March 2021; published 4 May 2021)

\begin{abstract}
Living systems at the subcellular, cellular, and multicellular levels are often crowded systems that contain active particles. The active motion of these particles can also propel passive particles, which typically results in enhanced effective diffusion of the passive particles. Here we study the diffusion of a passive tracer particle in such a dense system of active crowders using a minimal lattice model incorporating particles pushing each other. We show that the model exhibits several regimes of motility and quantify the enhanced diffusion as a function of density and activity of the active crowders. Moreover, we demonstrate an interplay of tracer diffusion and clustering of active particles, which suppresses the enhanced diffusion. Simulations of mixtures of passive and active crowders show that a rather small fraction of active particles is sufficient for the observation of enhanced diffusion.
\end{abstract}

DOI: 10.1103/PhysRevE.103.052601

\section{INTRODUCTION}

The behavior of individual particles within a crowded environment is influenced by the complex collective behavior of the surrounding particles [1-4]. Indeed, many biological systems are densely packed. Molecular systems in the cytoplasm of cells [1,5], colonies of bacteria [6], biofilms [7], and tissues [8] are a few examples. Often, the crowded environment contains active particles that are driven out of equilibrium due to the local injection and dissipation of energy. On the molecular scale, these active particles may be molecular motors pulling cargoes through cytoplasm and stirring it [9-11] or enzymes exhibiting enhanced diffusion [12,13]. On the cellular scale, these could be self-propelled cells swimming in a suspension or moving through a tissue or a colony of cells $[14,15]$. This means that detailed balance is not fulfilled in these systems, so they cannot be described by equilibrium statistical mechanics.

The observation of individual probe or tracer particles within such a system provides a method to quantify crowding effects as well as to probe for the degree of crowding if this is difficult to determine directly. Such probes include the diffusion of the tracer particle $[16,17]$ as well as specific chemical reactions or conformational changes such as the compaction of a polymer chain as measured by FRET-probes at the two ends of the polymer [18,19].

\footnotetext{
*Leila.abbaspour@theorie.physik.uni-goettingen.de

†Stefan.klumpp@phys.uni-goettingen.de
}

Published by the American Physical Society under the terms of the Creative Commons Attribution 4.0 International license. Further distribution of this work must maintain attribution to the author(s) and the published article's title, journal citation, and DOI.
Diffusion of molecules and particles in complex environments has been studied on a range of length scales and the complex environment can give rise to dynamic phenomena such as anomalous diffusion [20,21]. Specifically, diffusion of a passive tracer particle in an active system has been studied in various experimental and theoretical model systems. Notably, $\mathrm{Wu}$ and Libchaber [22] studied the trajectories of polystyrene beads in a suspension of swimming bacteria and observed enhanced diffusion of the beads. Such enhanced diffusion has been studied extensively, both theoretically [23-30] and experimentally [25,31-37]. Extensions of the simplest system include three-dimensional systems [36], the presence of obstacles [34] and confinement, and effective interaction between passive particles [38].

Interactions between particles in suspension can be complex and generally involve hydrodynamic interactions in addition to simple excluded volume and particle-particle collisions. Therefore, simplified minimal model systems can help to advance our understanding of the role of active processes. Both continuum models [39] and lattice models [40,41] have been used for that purpose. Enhanced diffusion of a tracer in a crowded active system has been observed in models with and without hydrodynamic interactions $[25,42]$.

Specifically, lattice models provide a conceptually simple and often computationally inexpensive approach to study complex phenomena based on particle-particle interactions. In the case of active particle systems, lattice approaches have been used to study clustering and phase separation [27,43-46]. Specifically, modifications of the dynamic rules can easily be implemented to investigate whether results are generic or specific to given dynamics.

Following this approach and applying it to tracer diffusion, here we study a minimal lattice model incorporating particleparticle collisions as well as different diffusion coefficients of different particle types to investigate the effect of activity and density of crowders on tracer diffusion, neglecting 
(a)

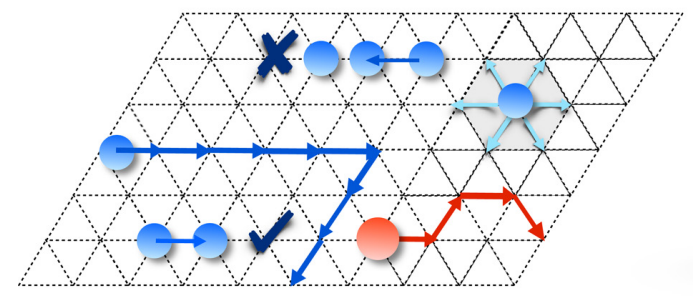

(c)

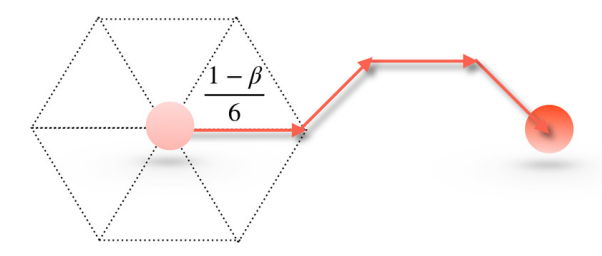

(b)

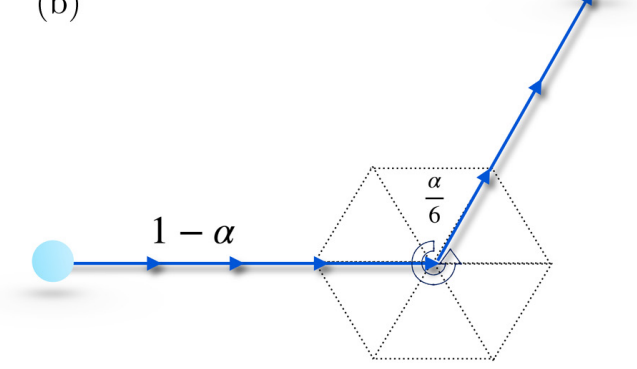

(d)

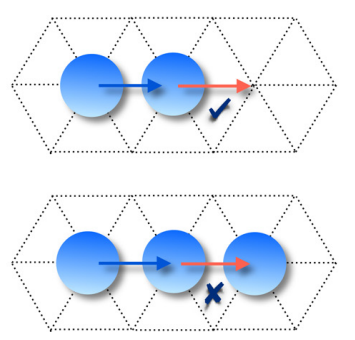

FIG. 1. Lattice model of a dense system of active particles (blue) containing a passive tracer particle (red). (a) Overview of the model on a hexagonal lattice. (b) Active particles perform run-and-tumble motion and move persistently in the same direction with probability $1-\alpha$ and randomize that direction (tumble) with probability $\alpha$ (i.e., $\alpha / 6$ for each direction). (c) The passive tracer particle (red) performs a simple random walk; its diffusion coefficient is modulated by the parameter $\beta$. (d) Collision rules among particles.

hydrodynamic interactions. Importantly, the collision rule we introduce goes beyond the simple exclusion rule often used in lattice models and allows for the pushing of one particle by another particle attempting to move to the first one's lattice site. We quantify the enhanced diffusion as a function of these parameters and relate the observed tracer diffusion to clustering of active particles. Finally, we generalize our analysis to the case of a binary mixture of active and passive particles. Our results indicate how the interplay between activity and density of crowders can lead to significant enhancement of the diffusion of a tracer but also limits that enhancement through clustering of the active particles.

\section{MODEL}

We consider a hexagonal lattice [see Fig. 1(a)] in two dimensions, consisting of $M=256 \times 256$ sites, with periodic boundary conditions (effects of the system size are discussed in Appendix A). There are in total $n$ particles on the lattice, of which $N_{a}=\chi_{A} N$ are active and can self-propel following a simplified run-and-tumble motion, and the rest are passive and exhibit diffusive behavior. We define $\rho$ as the number density of the system, which is defined as the total number of particles divided by the number of lattice sites.

For updating the position of all particles at each time step, $n$ random actions take place in the system. A particle may be picked more than once in one single time step, giving rise to fluctuation of its speed. On average, however, all particles are picked equally often.

The active particles have run-and-tumble dynamics, where they move persistently in one direction (initially chosen at random) and then reorient with a tumbling probability $\alpha$, resulting in long directed runs with abrupt turns. At each time step, the active particle moves in the same direction as in its last step with probability $1-\alpha$, and tumbles to choose a new direction with probability $\alpha / 6$. For instance, for $\alpha=$ 0.001 , the active particle takes, on average, 1000 persistent steps in the same direction, and then tumbles and chooses one out of the six possible directions with equal probability [see Fig. 1(b)]. In the case of passive particles, we set $\alpha=1$ : The particle reorients at each time step and therefore steps to each site with a probability of $1 / 6$. Since our aim is to study the effect of active particles on the overall motion of a passive particle, we put (at least) one passive tracer in the system, which in general has a different Brownian diffusion coefficient set by a parameter $\beta$. The tracer steps to each neighbor site with probability $(1-\beta) / 6$, with $0 \leqslant \beta \leqslant 1$ as shown in Fig. 1(c). When $\beta=0$, the tracer has the same dynamics as an ordinary passive particle, meaning it can move in each time step. As $\beta$ increases, the tracer diffuses more slowly and, in the limiting case of $\beta=1$, it can only move by being pushed by its neighbors.

We now define collision rules among the particles. We use a modified exclusion rule, which in addition to ensuring that each site is occupied by at most one particle also allows for particles pushing each other. In each stepping attempt of a particle, we check whether the target site on the lattice is empty or occupied. If it is empty, then the particle is allowed to update its position, as described above. If the site is occupied, then there are two possibilities, depending on the second-neighbor site of the particle in the same direction. If the latter site is empty, the stepping particle pushes the particle in the target site to the second-neighbor site and moves to the target site itself. Otherwise, the update is rejected [see Fig. 1(d)]. Note that there is no alignment interaction between particles in the pushing process. All simulations described in the following 


$$
\beta=0.0
$$

(a)

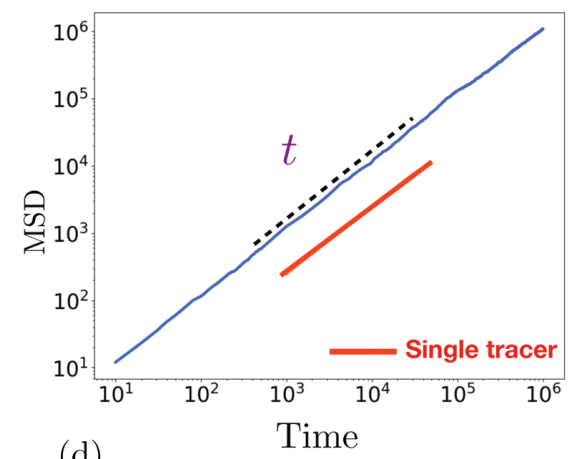

(d)

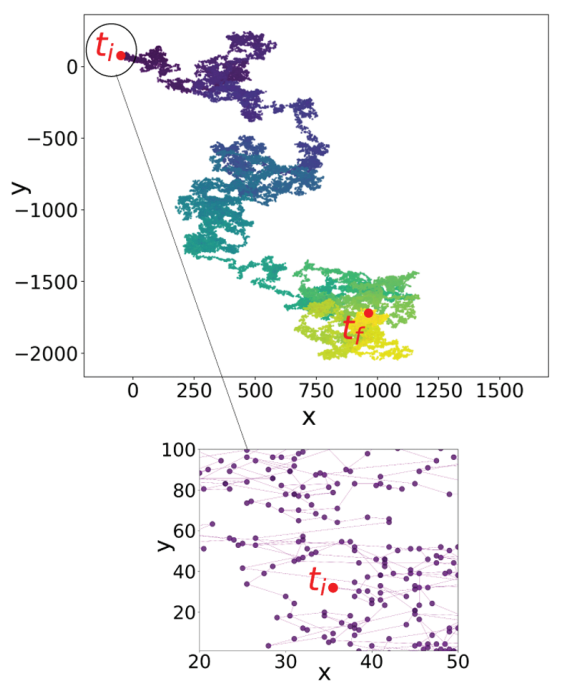

$\beta=0.9$

(b)

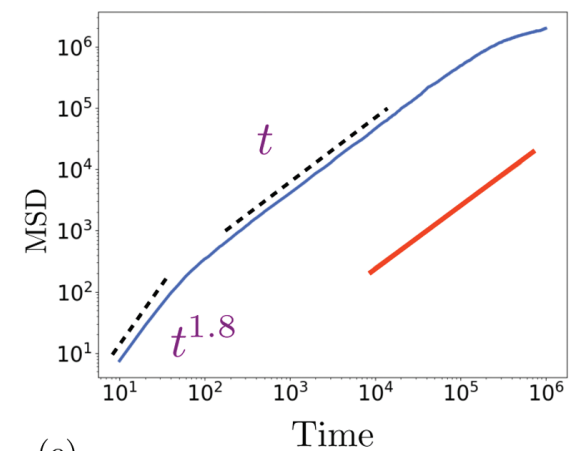

(e)

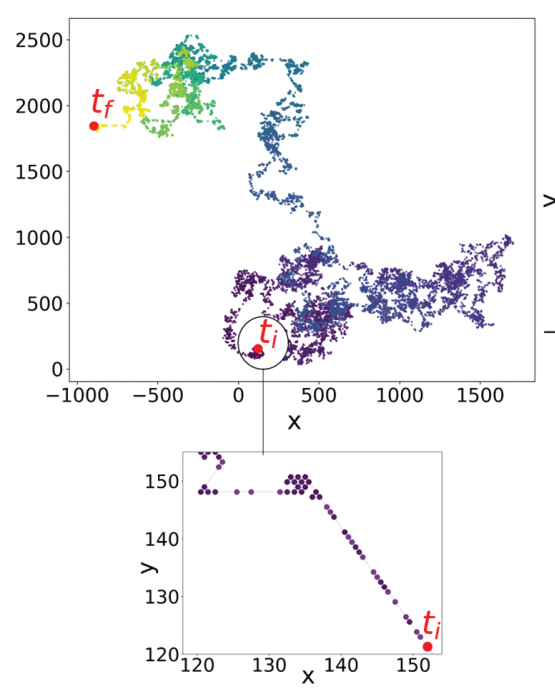

$\beta=1.0$

(c)

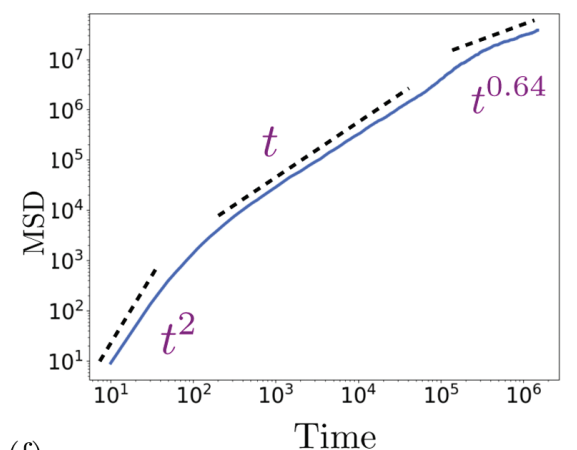

(f)
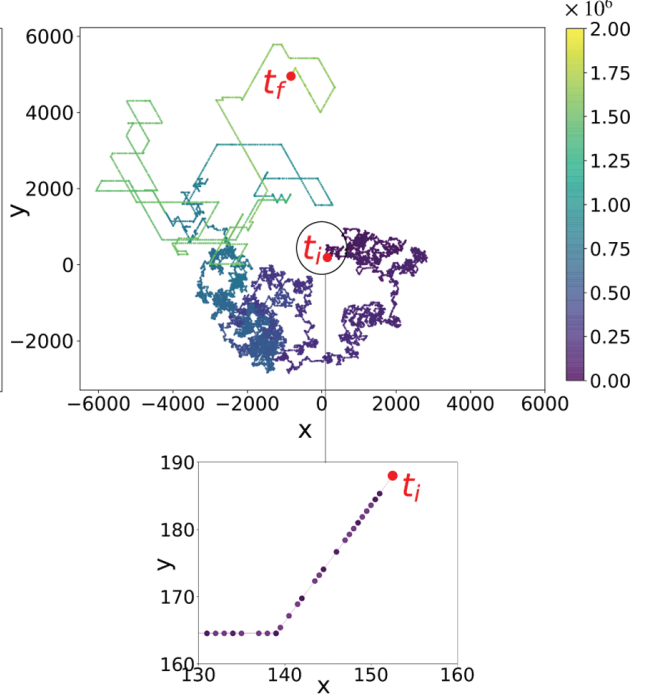

FIG. 2. Diffusion of tracer particles with different diffusion coefficients in a system of active crowders. (a)-(c) The mean-square displacement (thick blue lines) shows diffusive motion for $\beta=0$ and anomalous diffusion for $\beta=0.9,1$. The red lines indicate the diffusive motion of the tracer in an otherwise empty lattice in (a) and (b); in (c), the tracer would not move in that case. The dashed lines indicate the scaling behavior of the different regimes by showing the power laws fitted to the mean-square displacement. (d)-(f) show trajectories $\left(t_{i}\right.$ and $t_{f}$ indicate initial and final time, respectively, in the trajectory plots), the figures in the last row show the zoomed view of the trajectories at the early stages. Each column corresponds to the value of $\beta$ indicated above it. The other parameters are $\rho=0.025, \alpha=0.0001$.

were run for $10^{6}$ time steps with 1000 realizations of each condition.

\section{RESULTS}

\section{A. Tracer in a purely active system}

\section{Mean-square displacement}

We first discuss the results for a passive tracer particle in the presence of only active crowders (i.e., $N_{a}=N$ or $\chi_{A} \approx 1$ ). We considered densities in the range of $0.01 \leqslant \rho \leqslant 0.4$ and the tumbling probability of active particles between $0.0001 \leqslant$ $\alpha<1$.

We begin our analysis by evaluating the mean-square displacement (MSD) of the tracer particles. We note that for Brownian motion, the MSD is given by $\left\langle(\Delta r)^{2}\right\rangle=2 d D t$, where $\Delta r$ is the displacement of a particle in a given time interval of $t, d$ is the spatial dimension (which is 2 in our case), $D$ is the diffusion coefficient, and $\langle\cdot\rangle$ denotes an ensem- ble average. So-called anomalous diffusion is characterized by an MSD $\left\langle(\Delta r)^{2}\right\rangle \sim t^{\gamma}$, where $\gamma \neq 1$ is a real positive number that classifies the different types of diffusion: subdiffusion for $0<\gamma<1, \gamma=1$ is a typical diffusion process and super-diffusion for $\gamma>1$ (where $\gamma=2$ gives rise to ballistic motion).

In Figs. 2(a)-2(f), we have plotted the MSD and corresponding trajectories as a function of time for the three cases of $\beta=0,0.9$, and 1 . In all three cases, the density and the tumbling probability of crowders are the same: $\rho=0.025$ and $\alpha=0.0001$. For a purely passive Brownian tracer with $\beta=0$ [Figs. 2(a) and 2(d)], the tracer shows normal diffusion, with an MSD that is linear in time throughout the simulation. The trajectory is erratic at both long and short times [Fig. 2(d) and its inset, respectively].

It is useful to compare the diffusion coefficient of the tracer in this system to that of a passive tracer in the absence of active crowders. A general expression for the diffusion coefficient of a single passive particle on an arbitrary isotropic lattice 
(a)

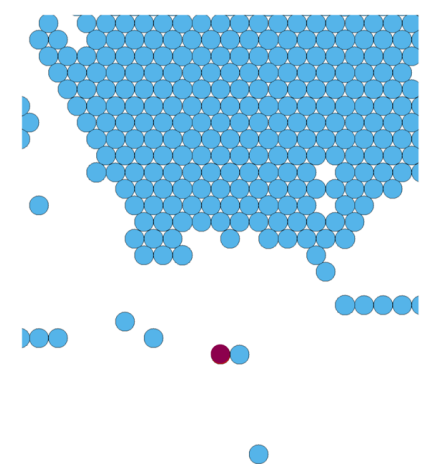

(b)

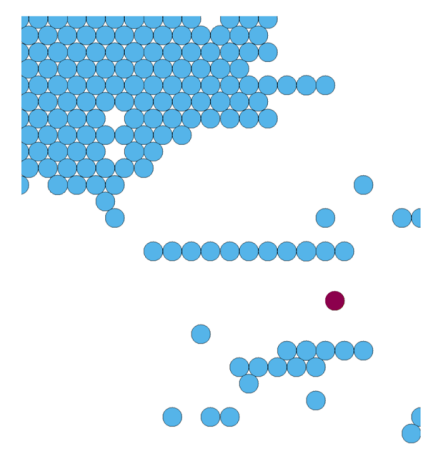

(c)

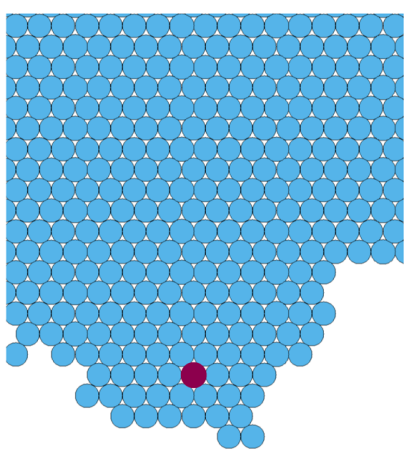

FIG. 3. Snapshots of the simulation for $\rho=0.1$ and $\alpha=0.0001$ : the active particles are shown in blue and the tracer particle in purple. (a) The tracer (purple) is next to an active particle (blue), which pushes it. (b) The tracer is trapped in empty space. (c) The tracer is trapped by active particles.

is $D_{0}=(\kappa / 2 d) a^{2} \lambda$, where $a$ is the lattice spacing (distance between neighboring lattice sites), $d$ is the spatial dimension, $\kappa$ is the coordination number (the number of neighbor sites allowed for a jump), and $\lambda$ is the stepping rate to the nearest neighbor site [47]. In our model $a=1$ (i.e., all lengths are expressed in units of the lattice spacing), $\kappa=6, d=2$, and $\lambda=\frac{1-\beta}{6}$, leading to

$$
D_{0}=\frac{1-\beta}{4} .
$$

Thus, for $\beta=0$, we find $D_{0}=1 / 4$. The corresponding MSD of a particle on an otherwise empty lattice is shown as the red line in Fig. 2(a). A comparison of the MSD without active crowders (red line) and the MSD in their presence (blue line) shows that the diffusion of our tracer particle is strongly enhanced by the active crowders.

For $\beta=0.9$ and $\beta=1$, by contrast, we can identify several diffusive regimes (indicated by the dashed lines, which show the scaling in the different regimes with behavior $\sim t^{\gamma}$ and $\gamma$ obtained by fitting). A slow tracer with $\beta=0.9$ initially undergoes superdiffusion [Fig. 2(b)] as it remains in front of an active particle over an extended period of time [see Fig. 3(a)]. The tracer inherits the direction of an individual active particle which drives it forward. This effect can also be seen in the inset of Fig. 2(e), in which this directed motion yields a straight line. The observed diffusion exponent $\gamma$ at short time is found to be 1.8, which is close to the ballistic regime with $\gamma=2$. After a longer time, diffusive motion is recovered, with erratic trajectories similar to those for $\beta=0$. The data also suggest that there may be a subdiffusive regime for very long times, see below.

Finally, Figs. 2(c) and 2(f) show the case where the passive tracer does not move by itself and only moves when pushed by an adjacent active particle. Initially, the tracer fully inherits the direction and the speed of the active particle that pushes it [Fig. 2(f), inset]. Since there is only self-propulsion, $\gamma$ is exactly 2 . For an intermediate time, diffusive behavior is recovered. Then, after some time, the motion becomes remarkably less random, exhibiting long directed paths and pauses, as depicted in the trajectory in Fig. 2(f). Correspondingly, the exponent of the MSD is reduced to $\gamma \sim 0.64$, indicating a subdiffusive behavior. Inspection of snapshots of the lattice [Figs. 3(b) and 3(c)] show that during the pauses, the tracer is either trapped in an empty region of the lattice and thus cannot be moved or in a cluster of active particles, within which it is also immobilized. This observation indicates that, in this case, the environment in which the tracer diffuses through changes from a homogeneous environment to coexistence of a dilute phase and clusters of active particles. Cluster formation will be discussed in more detail below.

\section{Neighbor site occupation and trapping}

To quantify the effect of trapping on the motion of a tracer, we define a trapping parameter $n$, which characterizes the occupation of the six nearest-neighbor sites of the tracer [see Fig. 4(a)]. Thus, $0 \leqslant n \leqslant 6$, with $n=0$ indicating that the tracer has no neighboring particle and $n=6$ is the fully trapped state. When $n=0$, the tracer with $\beta=1$ is essentially trapped by the empty space and becomes immobile, while tracers with $\beta=0$ and $\beta=0.9$ diffuse on their own. In the fully occupied state $(n=6)$, the tracer is immobile for all cases. $n=1$ results in persistent directed motion of the tracer if the neighboring active particle moves to the site of the tracer and $1-\beta<1-\alpha$, as the active particle then pushes the tracer for multiple steps.

For the two cases $\beta=0$ and $\beta=0.9$, the histograms of $n$ do not vary significantly over time, with the probability of having no neighbors being the largest [Figs. 4(b) and 4(c)]. As expected, the probability of having a single neighbor $(n=1)$ is higher for $\beta=0.9$ than $\beta=0$, in agreement with the observed directed motion. For $\beta=1, n=1$ is the most probable configuration at all times. However, the histogram remarkably broadens over time and becomes bimodal at long times, with $n=0$ and $n=6$ occurring (almost) equally frequently. This means that the three most likely configurations are those that lead to directed motion $(n=1)$, trapping in empty space $(n=0)$ or trapping in a cluster $(n=6)$, which together explain the range of motion observed in Fig. 2(f). This trapping appears to be the reason for the subdiffusion observed in this case for the long-time regime. In contrast, no such trapping is clearly seen for the case $\beta=0.9$. We therefore hypothesize that the apparent transition to subdiffusion at long times in this case may be due to more transient trapping of the tracer, 

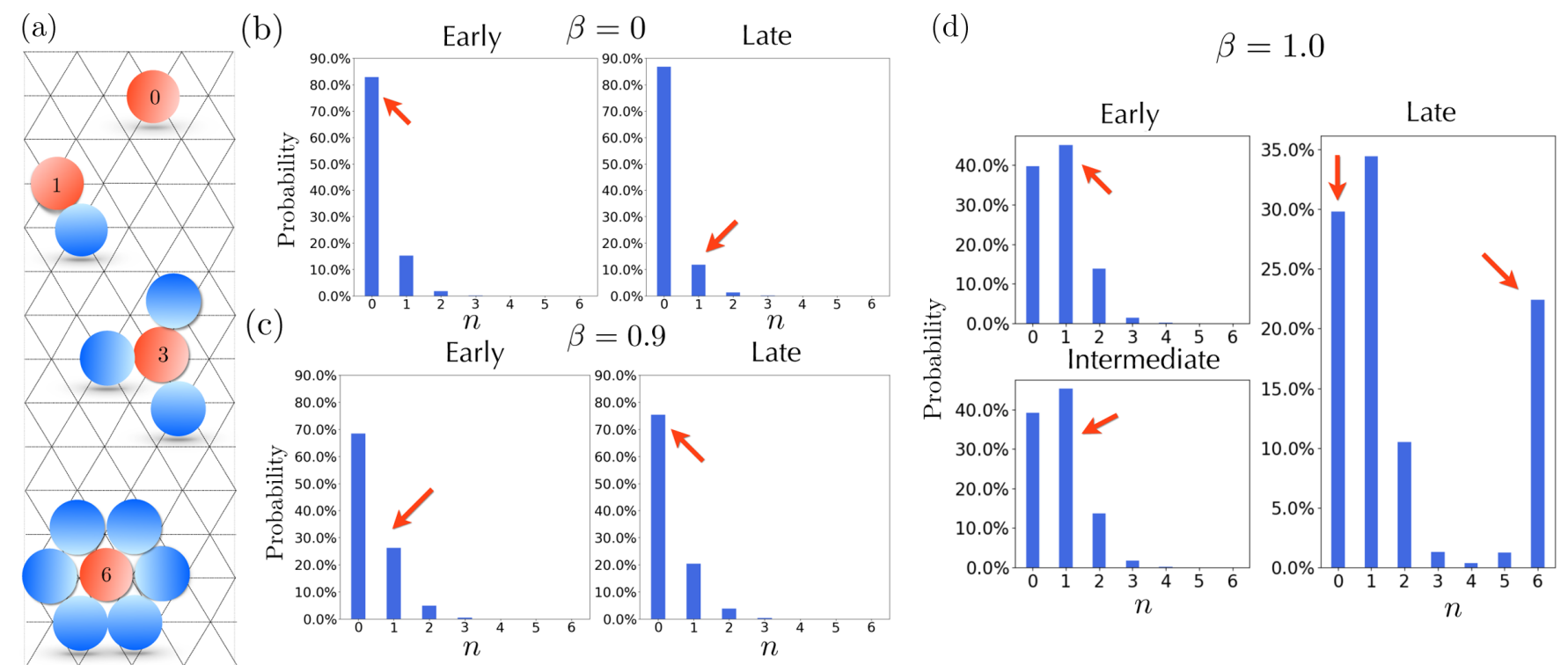

FIG. 4. Trapping analysis. (a) The sketch shows the tracer (red) surrounded by different number of active particles (blue). (b)-(d) show the probability that $n$ neighbor sites are occupied (for $n=0, \ldots 6$ ). The red arrows indicate the numbers of occupied neighbors most relevant for the different regimes in the MSD plots in Fig. 2. The probabilities were determined by averaging over 1000 simulation steps (starting at time 0 for the early state, at time 1000 for the intermediate state, and 1000 steps before time $10^{6}$ for the late state) as well as over 1000 realizations of the simulation (with random initial conditions).

e.g., between just two active particles moving into opposite directions.

\section{Effective diffusion coefficient at large times}

We have observed above that diffusion of the tracer is enhanced in the bath of active particles compared to the tracer on its own. To study this further, in Fig. 5, we plot the diffusion coefficient at large times as a function of density, focusing on the case $\beta=0.9$. The simulation was performed for a system with $\chi_{A}=1$ and different values of $\alpha$. Remarkably, the effective diffusion coefficient has a 50-fold increase when (a)

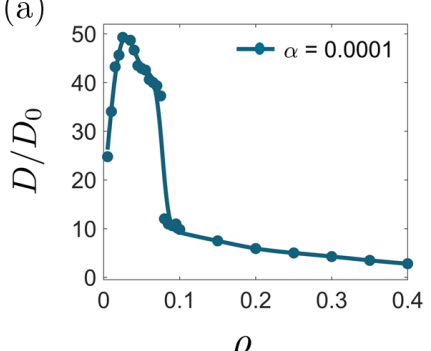

(c)

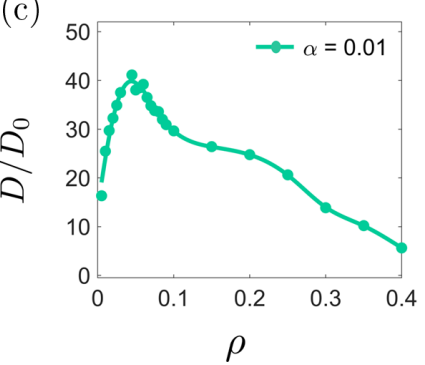

(b)

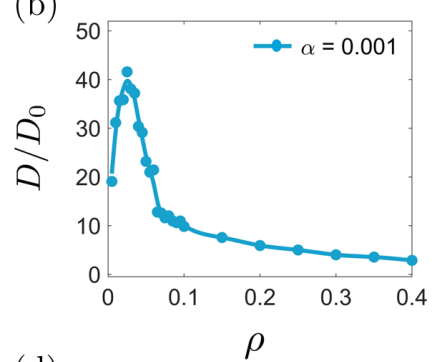

(d)

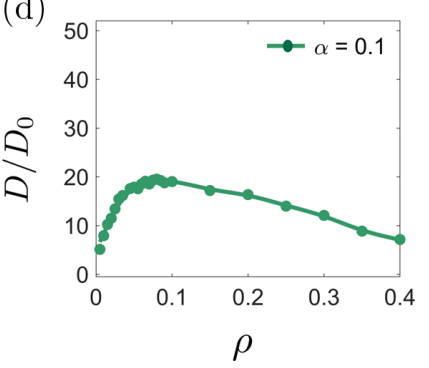

FIG. 5. The relative diffusion coefficient of a tracer with $\beta=0.9$, normalized to the diffusion constant $D_{0}$ of an isolated tracer. in the active bath, compared to the diffusion coefficient of an isolated tracer. Even at a low density $(\rho \approx 0.01)$ of active crowders, the diffusion coefficient of the tracer is substantially greater than in the absence of active crowders, implying a strong effect of being pushed by active particles.

Since the diffusion coefficient of a single passive tracer is a small constant in the absence of crowders and goes to zero in the presence of a high density of crowders, the relative diffusion coefficient is expected to be a nonmonotonic function of the density. Furthermore, the form of this dependence should also vary with the tumbling probability. The plots in Fig. 5 show a clear density dependence of the diffusion coefficient. The diffusion coefficient is sharply enhanced when the density of active particles is increased from zero, then decreases after a pronounced peak. Clearly, the density of the system greatly affects the diffusion coefficient of the tracer. To further investigate this effect, we come back to the observation of cluster formation. Additional analysis for the case $\beta=1.0$ is provided in Appendix B.

\section{Cluster formation}

To analyze clustering, we define a cluster as a collection of at least six particles sticking together, i.e., being connected via mutual nearest neighbors. Particles without nearest neighbors or connected to fewer particles are considered as belonging to the gas phase. Clusters are identified with the data analysis framework FREUD [48]. We measured the average cluster size, $C_{s}$, as the average number of particles in a cluster over 1000 different realizations of the simulation. Figures 6(a) and 6(b) show the time evolution of $C_{s}$ for different densities and for $\alpha=0.001$ and 0.0001 , respectively. As can be seen in Figs. 6(a) and 6(b), cluster formation is accelerated by increasing the density of crowders. 
(a)

$$
\alpha=0.001
$$

$$
\alpha=0.0001
$$

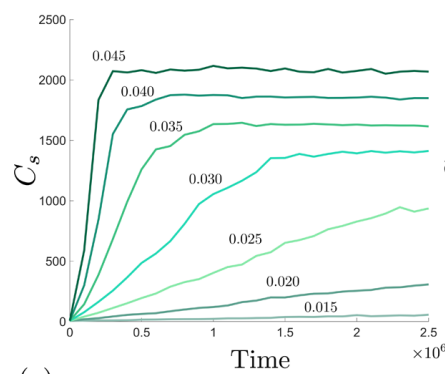

(c)

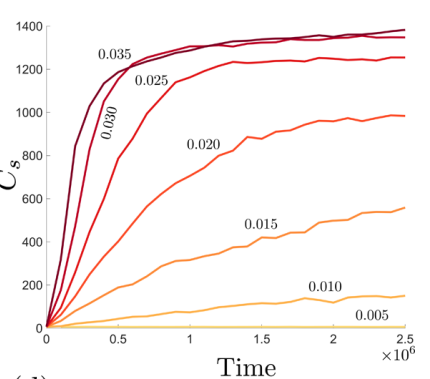

(d)

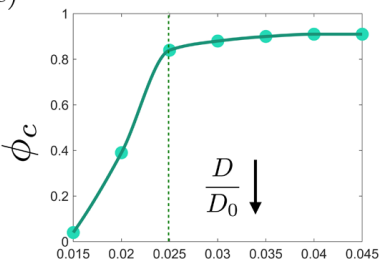

$\rho$

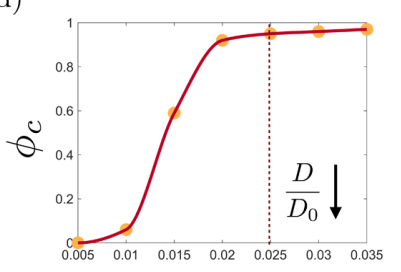

$\rho$

FIG. 6. Cluster analysis: (a), (b) Average cluster size as a function of time (averaged over 1000 realizations) for different densities. The numbers above the curves indicate the corresponding density. (c), (d) Fraction of particles assembled in clusters in the last snapshot of simulation for the same densities. The dashed lines mark the density in which the tracer exhibits the maximum diffusion coefficient. Each column corresponds to the value of $\alpha$ indicated above it.

In Figs. 6(c) and 6(d), we plotted the fraction of particles detected within clusters, $\phi_{c}$, as seen in the last snapshot of the simulation, which provides an estimate of the fraction of particles assembled in the clusters. $\phi_{c}$ can be described by $\left(N_{c} \times C_{s}\right) / N$, where $N_{c}$ is the average number of clusters. As a function of the density, $\phi_{c}$ shows a clear transition from a state in which a very small fraction of particles are in the clusters to a state where almost all particles are in clusters.

To relate clustering to the tracer diffusion discussed before, we indicate with the vertical dashed lines the density at which the tracer's diffusion coefficient for the given tumbling probability is maximal. These lines fall near or slightly above the transition, suggesting an intimate relation of the decline in diffusion to the onset of phase separation. When the active crowders start to form clusters, there is coexistence of a dilute gas phase and clusters of active particles and the probability of active crowders being in the dilute phase decreases as long as the cluster size increases. Consequently, the diffusion coefficient of the tracer decreases as there are fewer and fewer active crowders in the dilute phase to push the tracer and enhance its diffusion.

\section{B. Binary mixture}

Finally, we consider a binary mixture of active and passive crowders, with $\chi_{A}+\chi_{P}=1$, where $\chi_{P}$ is the fraction of passive particles. We tracked the effect of the three control parameters $\chi_{A}, \alpha$, and $\rho$ on the diffusion coefficient of a tracer particle with $\beta=0.9$. Adding passive particles into a purely active system changes not only the directionality imposed by collisions (due to pushing by the active particles), but also

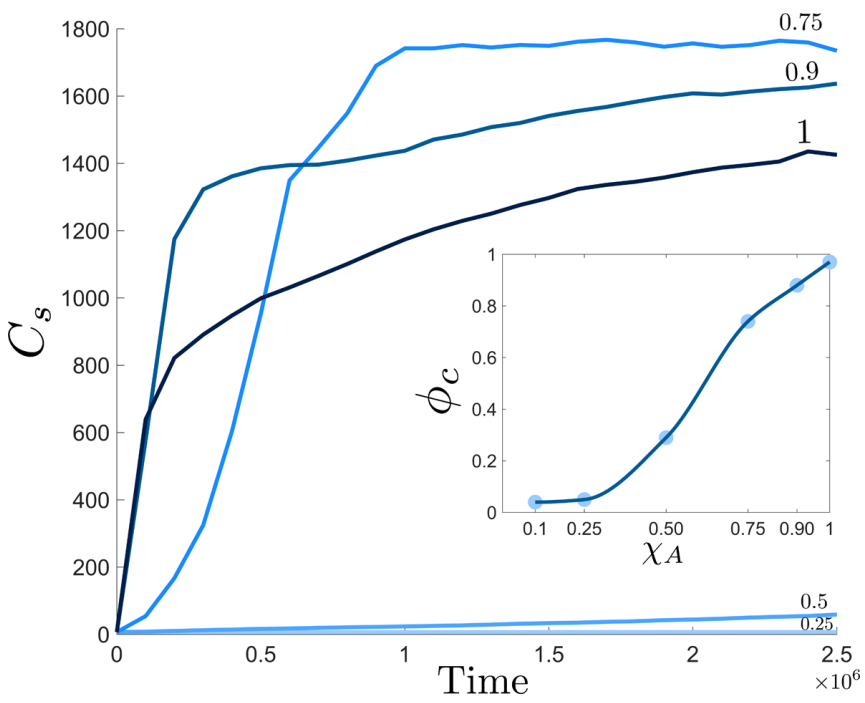

FIG. 7. Cluster analysis for different fractions $\chi_{A}$ of active particles at fixed $\rho=0.05$. Cluster size and fraction of particles in clusters (inset) as in Fig. 6 for different $\chi_{A}$ in a binary mixture of active and passive crowders. The numbers above each curve indicate the fraction of active particles $\left(\chi_{A}\right)$.

alters the global dynamics in a subtle way, specifically the dynamics of clustering. In the example shown in Fig. 7 with a fixed low density of crowders, an increase of the fraction of active crowders above 0.5 leads to a strong clustering signature both in the average cluster size $C_{s}$ and in the fraction $\phi_{c}$ of particles within clusters (inset). The cluster size $C_{s}$ shows biphasic growth for large fractions of active crowders with, first, a rapid increase in the cluster size and then a much slower second phase, which appears to be more pronounced and slower for the largest active crowder fractions. Put differently, replacing some of the active crowders with passive particles delays the phase separation (compare the cases $\chi_{A}=1$ and $\chi_{A}=0.75$ in Fig. 7), likely because, initially, the rapid directional change of the passive particles randomizes the motion of the active particles. Eventually, however, clustering sets in. While clustering sets in later for $\chi_{A}=0.75$ than for $\chi_{A}=1$, it reaches its stationary state faster.

Above we have shown a correspondence between the onset of clustering and a decrease of the enhanced diffusion of a passive tracer (Fig. 5). Since clustering is decreased by increasing the fraction of passive particles in the system, we expect that the decrease in diffusion of the tracer is less pronounced when passive particles are present. This is indeed the case, as shown in Fig. 8, where we plot the diffusion coefficient as a function of the density for different combinations of the tumbling probability $\alpha$ and the fraction of active crowders $\chi_{A}$ : Decreasing the fraction of active crowders leads to a broader peak in the diffusion coefficient as a function of density. We interpret this observation as reflecting the fact that at low $\chi_{A}$, it is more likely to find many of the active particles (which can push the tracer) in the gas phase compared to higher $\chi_{A}$. At the same time, however, the value of the maximal diffusion coefficient is also decreased, reflecting the fact that the total number of active particles is reduced. Nevertheless, even for very low fractions of active particles (bottom row in Fig. 8), 


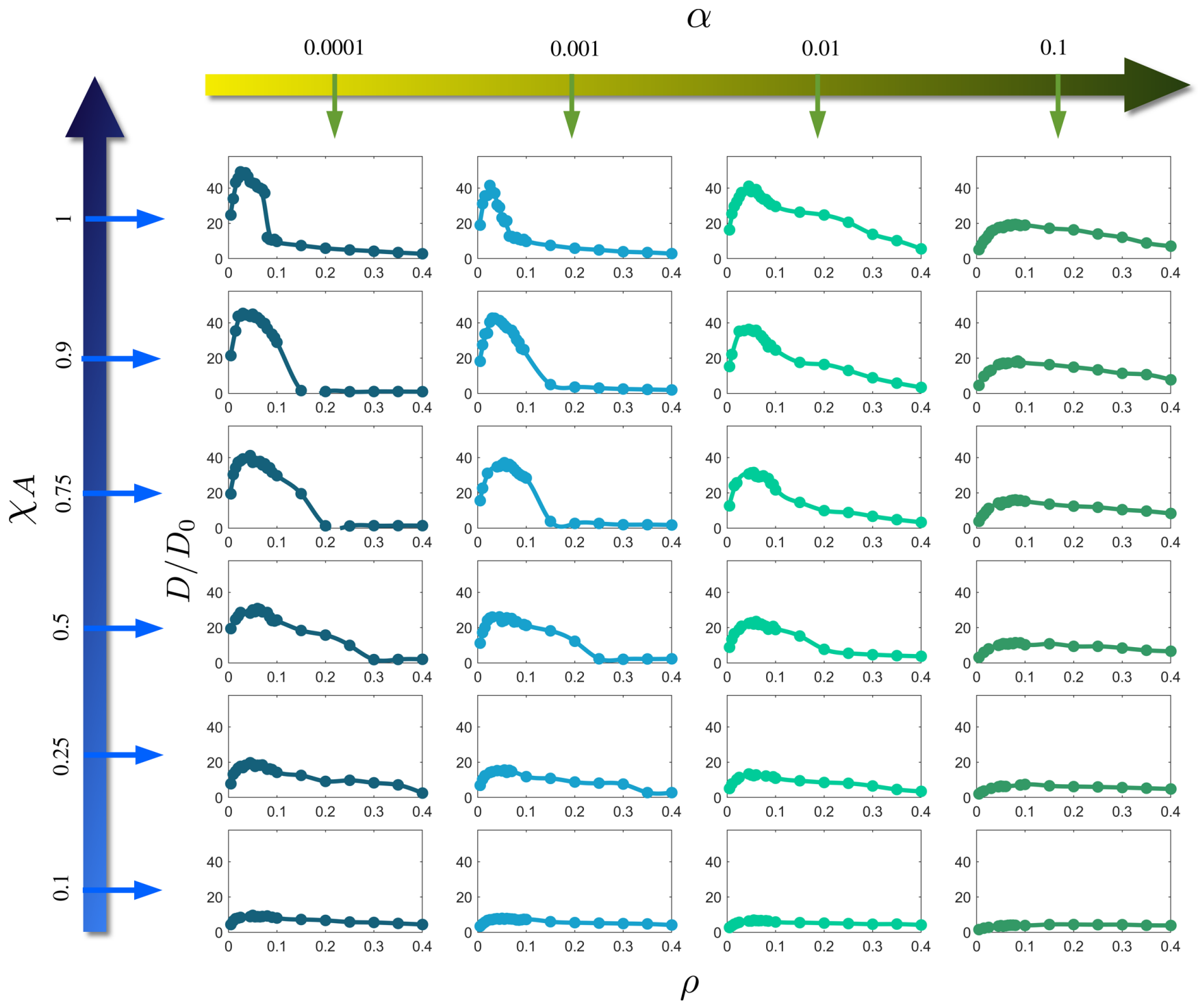

FIG. 8. The diffusion coefficient of a tracer with $\beta=0.9$ as a function of the density $\rho$ and of the fraction of active row $\chi_{A}$ in a binary mixture.

the diffusion coefficient is still increased compared to the case with only passive particles. For instance, for $\alpha=0.1$, $\chi_{A}=0.1$, it is increased up to fivefold.

\section{CONCLUSION}

In this paper, we have analyzed the diffusion of a tracer particle in a crowded active system using a minimal lattice model. We implemented the pushing of a passive tracer by active crowder particles by a simple collision rule. A passive tracer in an otherwise purely active system or in a binary mixture of active and passive particles exhibits different motility regimes, including significantly enhanced effective diffusion. Enhanced diffusion of tracers in the presence of active particles has been observed in several different systems such as in a bath of swimming microorganisms [22,25,33] and in a suspension of actively diffusing enzymes [13]. The activity mechanisms and the interaction in these systems are more complicated than those studied in our model, but the results shown here and related results of others [42] demonstrate that simple interactions are sufficient to generate such enhanced diffusion and can result in rich dynamic behavior. Our results show that the extent to which diffusion is enhanced depends on the activity of the crowder particles (modulated via their hopping rate relative to the tracer's hopping rate and via their tumbling probability, i.e., via the persistence of their motion) and on their density. The interplay of activity and density depends on the dynamics of the particles directly, but also indirectly via the phase separation of the crowders into lowdensity (gas-phase) regions and dense clusters. The latter has a negative effect on the mobility of the tracer, both when the tracer becomes trapped in a cluster and when the tracer moves in a low-density area, where it is not pushed by active particles. Our findings thus point to an intricate interplay between the local dynamics of enhanced tracer diffusion and the global dynamics of the system. 
One feature of active particle systems that we have not considered here are hydrodynamic interactions, which are typically present. On the one hand, studies like our present one or Ref. [49] show that phenomena such as enhanced tracer diffusion that are often observed experimentally in systems, where hydrodynamic interactions are present, are not dependent on those hydrodynamic interactions but can also be found in reduced models without them. However, in general, one can expect that the quantitative results are modified by the inclusion of hydrodynamic interactions, both between the particles and with the surfaces that confine the particles to a quasi-two-dimensional system [50-52]. We hypothesize that the dominant effect of hydrodynamic interactions would be due to the interactions of particles with the surface of relatively rigid clusters of particles and results in a rotation of the arriving particle, similar to the case of a rigid wall. Depending on the extent of the resulting torque, such interactions could enhance or reduce cluster formation. One way of including such effects into a minimal lattice model would be via an additional update rule that rotates particles near the surface of a cluster.

Another aspect that could be of interest is whether the underlying lattice type influences the dynamics of the tracer particle. Phase separation as observed here has indeed been studied in models of active particles on different lattices [44,46]. Specifically, different lattices may allow different interaction rules, particularly if, as in our case, lattice sites beyond the nearest neighbor have an influence. For example, one recent study showed clustering and a hexatic phase in a system of passive particles on a hexagonal lattice [45].

Given the simplicity of our model, one can also extend it to other collision rules among the particles as well as use it to study the impact of active crowding on physical processes other than tracer diffusion.

\section{ACKNOWLEDGMENTS}

This research was conducted within the Max Planck School Matter to Life, supported by the German Federal Ministry of Education and Research (BMBF) in collaboration with the Max Planck Society. The authors acknowledge further support by DFG through SFB 937 Project No. A21 in the initial phase of this work. The simulations were run on the Goegrid cluster at the University of Göttingen, which is supported by the DFG (Grant No. INST 186/1353-1 FUGG) and MWK Niedersachsen (Grant No. 45-10-19-F-02).

\section{APPENDIX A: EFFECT OF SYSTEM SIZE}

To check whether the size of the lattice affects the results presented here, we ran simulations with different system sizes $(L=50,100,256,512)$. Figure 9 shows the MSD of the tracer particle for two different densities of active crowders
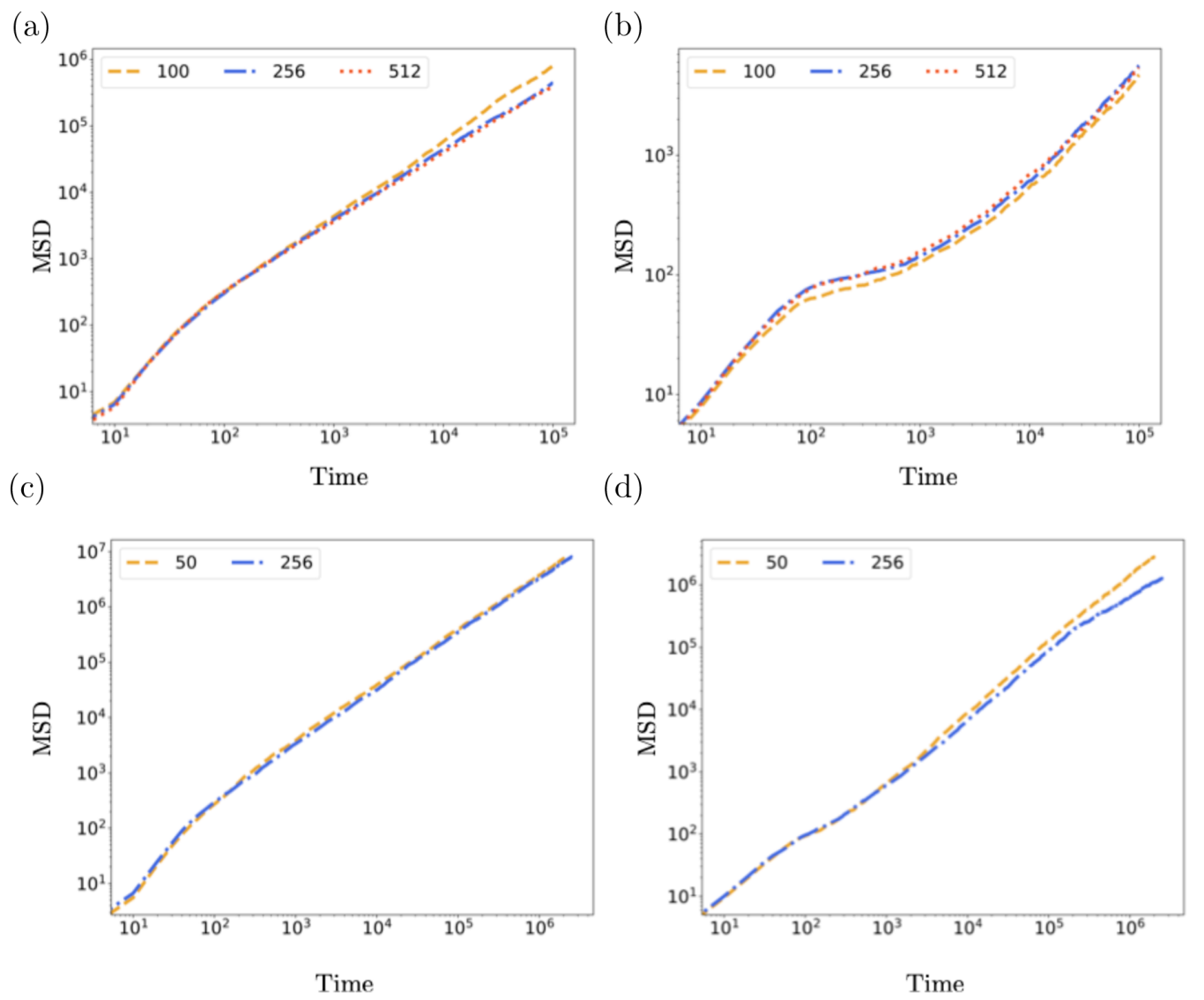

FIG. 9. MSD as a function of time plotted for different simulation box size $(L=50,100,256$ and 512$)$ for densities (a) $\rho=0.02$ and (b) $\rho=0.4$, both with $\alpha=0.0001$ (c) $\rho=0.02$ and (d) $\rho=0.4$, both with $\beta=0.9$ and $\alpha=0.01$. $\beta=0.9$ in all cases. All the plots are averaged over 1000 realizations for each box size. 
(a)

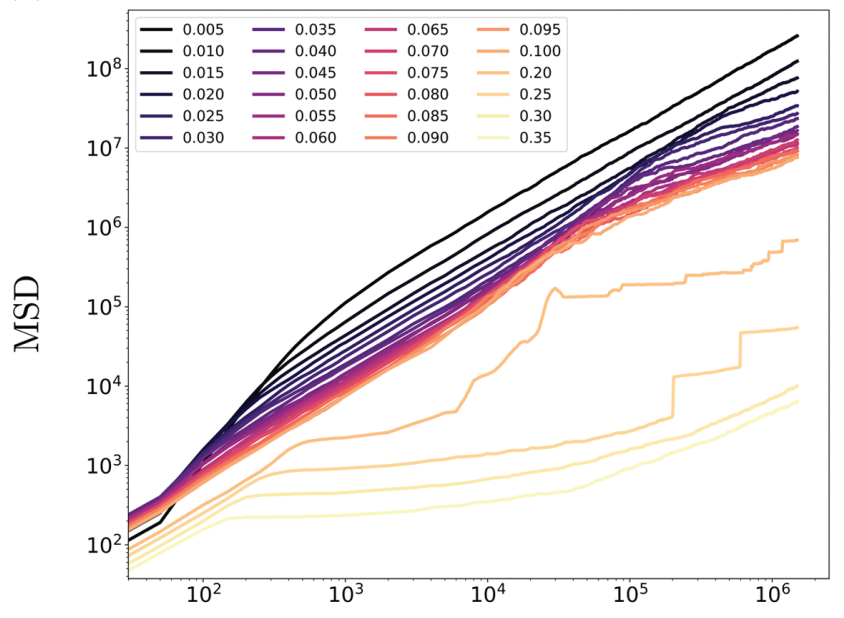

(c)

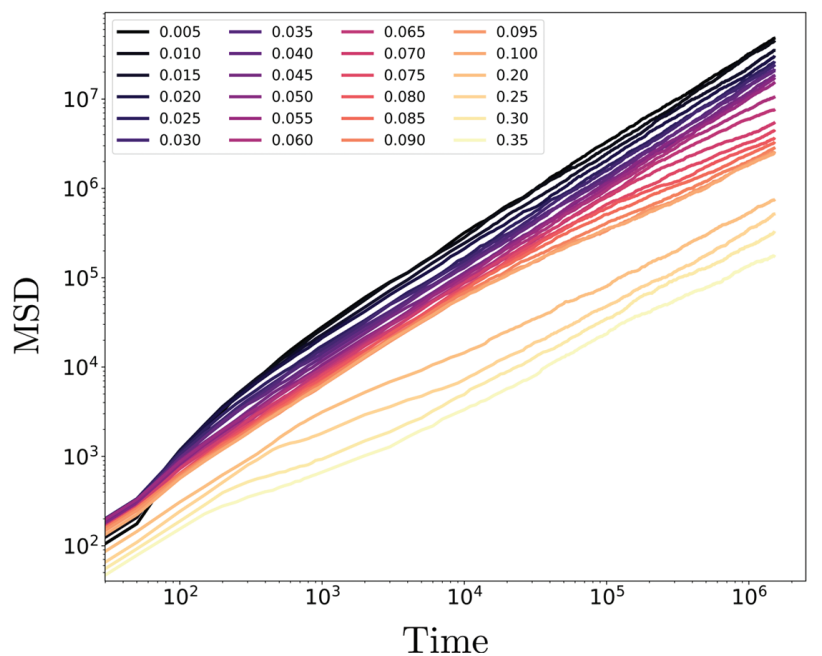

(b)

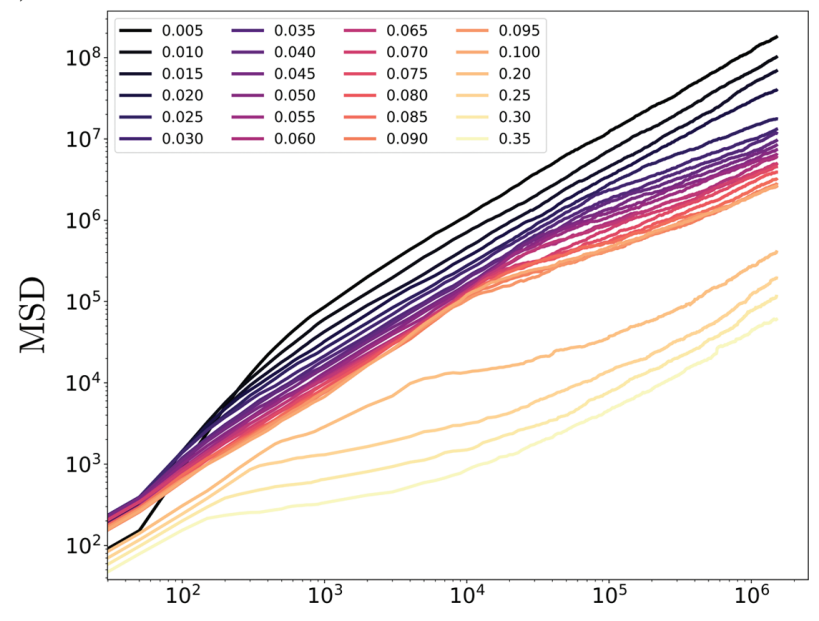

(d)

Time

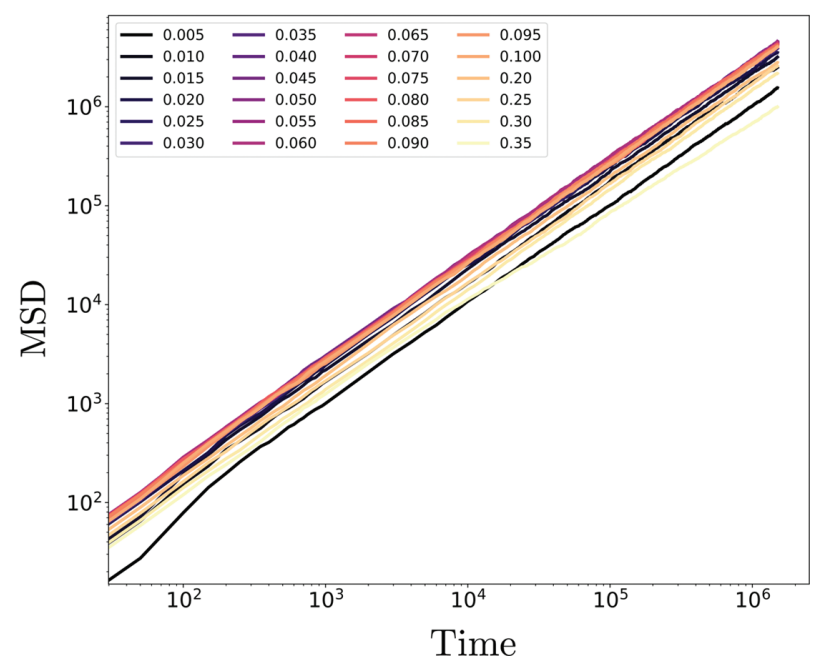

FIG. 10. The mean-square displacement of a tracer with $\beta=1.0$ as a function of time for different densities and tumbling probabilities (a) $\alpha=0.0001$, (b) $\alpha=0.001$, (c) $\alpha=0.01$, (d) $\alpha=0.1$.

( $\rho=0.02$ and $\rho=0.40)$. The results for different system sizes lie almost on top of each other, with only some deviations at long times for the smallest system size $[L=100$ in Fig. 9(a) and $L=50$ in Fig. 9(d)]. Importantly, while the small values of $\alpha$ used in many of our simulations in principle allow for persistent motion of the active particles over distances exceeding the system size, due to the interactions between the particles this is typically not the case except for very low densities.

\section{APPENDIX B: LONG-TIME DYNAMICS FOR $\boldsymbol{\beta}=1.0$}

The observation of trapping of the tracer in either clusters of active particles or in empty areas of the lattice in the case where the tracer cannot move on its own $(\beta=1)$ suggests the question whether such trapping and the subdiffusion resulting from it exhibits a sensitive dependence on the density, as a sufficient density may be required for trapping. We therefore simulated tracer mobility over a wide range of densities for $\beta=1$ and four different values of $\alpha$. As one can observe in Fig. 10, the tracer shows purely normal diffusion for the largest value of $\alpha$, with nonmonotonic dependence of the diffusion coefficient on density similar to Fig. 5. For the smaller values of $\alpha$, a subdiffusive regime appears for long times, as the active particles start to form stable or transiently stable clusters and trapping of the tracer starts to play a role. The transition to subdiffusion appears to occur at a critical density, which depends on the tumbling probability which is $\rho \simeq 0.020$ for $\alpha=0.0001, \rho \simeq 0.025$ for $\alpha=0.001$, and $\rho \simeq 0.060$ for $\alpha=0.01$. In all cases, these values fall near to the onset of phase separation as shown in Figs. 6(c) and 6(d). There also appears to be a second transition, clearly visible for $\alpha=0.001$ and $\alpha=0.01$, where for densities $\geqslant 0.25$ a plateau at intermediate times develops, likely also due to trapping. 
[1] R. J. Ellis, Macromolecular crowding: obvious but underappreciated, Trends Biochem. Sci. 26, 597 (2001).

[2] J. A. Dix and A. Verkman, Crowding effects on diffusion in solutions and cells, Annu. Rev. Biophys. 37, 247 (2008).

[3] C. Bechinger, R. Di Leonardo, H. Löwen, C. Reichhardt, G. Volpe, and G. Volpe, Active particles in complex and crowded environments, Rev. Mod. Phys. 88, 045006 (2016).

[4] S. Klumpp, W. Bode, and P. Puri, Life in crowded conditions, Eur. Phys. J.: Spec. Top. 227, 2315 (2019).

[5] S. B. Zimmerman and A. P. Minton, Macromolecular crowding: Biochemical, biophysical, and physiological consequences, Annu. Rev. Biophys. Biomol. Struct. 22, 27 (1993).

[6] N. H. Mendelson, A. Bourque, K. Wilkening, K. R. Anderson, and J. C. Watkins, Organized cell swimming motions in bacillus subtilis colonies: Patterns of short-lived whirls and jets, J. Bacteriol. 181, 600 (1999).

[7] L. Hall-Stoodley, J. W. Costerton, and P. Stoodley, Bacterial biofilms: From the natural environment to infectious diseases, Nat. Rev. Microbiol. 2, 95 (2004).

[8] G. Salbreux and F. Jülicher, Mechanics of active surfaces, Phys. Rev. E 96, 032404 (2017).

[9] D. Mizuno, C. Tardin, C. F. Schmidt, and F. C. MacKintosh, Nonequilibrium mechanics of active cytoskeletal networks, Science 315, 370 (2007).

[10] M. J. I. Muller, S. Klumpp, and R. Lipowsky, Tug-of-war as a cooperative mechanism for bidirectional cargo transport by molecular motors, Proc. Natl. Acad. Sci. USA 105, 4609 (2008).

[11] C. B. Korn, S. Klumpp, R. Lipowsky, and U. S. Schwarz, Stochastic simulations of cargo transport by processive molecular motors, J. Chem. Phys. 131, 245107 (2009).

[12] R. Golestanian, Anomalous Diffusion of Symmetric and Asymmetric Active Colloids, Phys. Rev. Lett. 102, 188305 (2009).

[13] X. Zhao, K. K. Dey, S. Jeganathan, P. J. Butler, U. M. CórdovaFigueroa, and A. Sen, Enhanced diffusion of passive tracers in active enzyme solutions, Nano Lett. 17, 4807 (2017).

[14] A. Sokolov and I. S. Aranson, Physical Properties of Collective Motion in Suspensions of Bacteria, Phys. Rev. Lett. 109, 248109 (2012).

[15] J. Dunkel, S. Heidenreich, K. Drescher, H. H. Wensink, M. Bär, and R. E. Goldstein, Fluid Dynamics of Bacterial Turbulence, Phys. Rev. Lett. 110, 228102 (2013).

[16] M. C. Konopka, K. A. Sochacki, B. P. Bratton, I. A. Shkel, M. T. Record, and J. C. Weisshaar, Cytoplasmic protein mobility in osmotically stressed escherichia coli, J. Bacteriol. 191, 231 (2009).

[17] B. R. Parry, I. V. Surovtsev, M. T. Cabeen, C. S. O’Hern, E. R. Dufresne, and C. Jacobs-Wagner, The bacterial cytoplasm has glass-like properties and is fluidized by metabolic activity, Cell 156, 183 (2014).

[18] A. J. Boersma, I. S. Zuhorn, and B. Poolman, A sensor for quantification of macromolecular crowding in living cells, Nat. Methods 12, 227 (2015).

[19] D. Gnutt, M. Gao, O. Brylski, M. Heyden, and S. Ebbinghaus, Excluded-volume effects in living cells, Angew. Chem. Int. Ed. 54, 2548 (2015).

[20] D. L. Koch and J. F. Brady, Anomalous diffusion in heterogeneous porous media, Phys. Fluids 31, 965 (1988).

[21] S. K. Ghosh, A. G. Cherstvy, D. S. Grebenkov, and R. Metzler, Anomalous, non-Gaussian tracer diffusion in crowded two-dimensional environments, New J. Phys. 18, 013027 (2016).

[22] X.-L. Wu and A. Libchaber, Particle Diffusion in a Quasi-TwoDimensional Bacterial Bath, Phys. Rev. Lett. 84, 3017 (2000).

[23] G. Grégoire, H. Chaté, and Y. Tu, Active and passive particles: Modeling beads in a bacterial bath, Phys. Rev. E 64, 011902 (2001).

[24] I. M. Zaid, J. Dunkel, and J. M. Yeomans, Lévy fluctuations and mixing in dilute suspensions of algae and bacteria, J. R. Soc. Interface 8, 1314 (2011).

[25] C. Valeriani, M. Li, J. Novosel, J. Arlt, and D. Marenduzzo, Colloids in a bacterial bath: Simulations and experiments, Soft Matter 7, 5228 (2011).

[26] D. O. Pushkin and J. M. Yeomans, Fluid Mixing by Curved Trajectories of Microswimmers, Phys. Rev. Lett. 111, 188101 (2013).

[27] R. Soto and R. Golestanian, Run-and-tumble dynamics in a crowded environment: Persistent exclusion process for swimmers, Phys. Rev. E 89, 012706 (2014).

[28] T. Kasyap, D. L. Koch, and M. Wu, Hydrodynamic tracer diffusion in suspensions of swimming bacteria, Phys. Fluids 26, 081901 (2014).

[29] A. Morozov and D. Marenduzzo, Enhanced diffusion of tracer particles in dilute bacterial suspensions, Soft Matter 10, 2748 (2014).

[30] Y. Yang and M. A. Bevan, Cargo capture and transport by colloidal swarms, Sci. Adv. 6, eaay7679 (2020).

[31] A. Caspi, R. Granek, and M. Elbaum, Enhanced Diffusion in Active Intracellular Transport, Phys. Rev. Lett. 85, 5655 (2000).

[32] M. J. Kim and K. S. Breuer, Enhanced diffusion due to motile bacteria, Phys. Fluids 16, L78 (2004).

[33] K. C. Leptos, J. S. Guasto, J. P. Gollub, A. I. Pesci, and R. E. Goldstein, Dynamics of Enhanced Tracer Diffusion in Suspensions of Swimming Eukaryotic Microorganisms, Phys. Rev. Lett. 103, 198103 (2009).

[34] G. Miño, T. E. Mallouk, T. Darnige, M. Hoyos, J. Dauchet, J. Dunstan, R. Soto, Y. Wang, A. Rousselet, and E. Clement, Enhanced Diffusion Due to Active Swimmers at a Solid Surface, Phys. Rev. Lett. 106, 048102 (2011).

[35] G. Mino, J. Dunstan, A. Rousselet, E. Clément, and R. Soto, Induced diffusion of tracers in a bacterial suspension: theory and experiments, J. Fluid Mech. 729, 423 (2013).

[36] A. Jepson, V. A. Martinez, J. Schwarz-Linek, A. Morozov, and W. C. K. Poon, Enhanced diffusion of nonswimmers in a three-dimensional bath of motile bacteria, Phys. Rev. E 88, 041002(R) (2013).

[37] N. Koumakis, A. Lepore, C. Maggi, and R. Di Leonardo, Targeted delivery of colloids by swimming bacteria, Nat. Commun. 4, 1 (2013).

[38] L. Angelani, C. Maggi, M. L. Bernardini, A. Rizzo, and R. Di Leonardo, Effective Interactions between Colloidal Particles Suspended in a Bath of Swimming Cells, Phys. Rev. Lett. 107, 138302 (2011).

[39] S. K. Ghosh, A. G. Cherstvy, and R. Metzler, Non-universal tracer diffusion in crowded media of non-inert obstacles, Phys. Chem. Chem. Phys. 17, 1847 (2015).

[40] O. Bénichou, P. Illien, G. Oshanin, A. Sarracino, and R. Voituriez, Tracer diffusion in crowded narrow channels, J. Phys.: Condens. Matter 30, 443001 (2018). 
[41] C. Mejia-Monasterio, S. Nechaev, G. Oshanin, and O. Vasilyev, Tracer diffusion on a crowded random manhattan lattice, New J. Phys. 22, 033024 (2020).

[42] E. W. Burkholder and J. F. Brady, Tracer diffusion in active suspensions, Phys. Rev. E 95, 052605 (2017).

[43] J. T. Siebert, F. Dittrich, F. Schmid, K. Binder, T. Speck, and P. Virnau, Critical behavior of active Brownian particles, Phys. Rev. E 98, 030601(R) (2018).

[44] S. Whitelam, K. Klymko, and D. Mandal, Phase separation and large deviations of lattice active matter, J. Chem. Phys. 148, 154902 (2018).

[45] S. Darjani, J. Koplik, S. Banerjee, and V. Pauchard, Liquidhexatic-solid phase transition of a hard-core lattice gas with third neighbor exclusion, J. Chem. Phys. 151, 104702 (2019).

[46] B. Partridge and C. F. Lee, Critical Motility-Induced Phase Separation Belongs to the Ising Universality Class, Phys. Rev. Lett. 123, 068002 (2019).
[47] O. M. Braun and C. A. Sholl, Diffusion in generalized latticegas models, Phys. Rev. B 58, 14870 (1998).

[48] V. Ramasubramani, B. D. Dice, E. S. Harper, M. P. Spellings, J. A. Anderson, and S. C. Glotzer, freud: A software suite for high throughput analysis of particle simulation data, Comput. Phys. Commun. 254, 107275 (2020).

[49] A. Mozaffari, N. Sharifi-Mood, J. Koplik, and C. Maldarelli, Self-propelled colloidal particle near a planar wall: A Brownian dynamics study, Phys. Rev. Fluids 3, 014104 (2018).

[50] K. Schaar, A. Zöttl, and H. Stark, Detention Times of Microswimmers Close to Surfaces: Influence of Hydrodynamic Interactions and Noise, Phys. Rev. Lett. 115, 038101 (2015).

[51] A. Mozaffari, N. Sharifi-Mood, J. Koplik, and C. Maldarelli, Self-diffusiophoretic colloidal propulsion near a solid boundary, Phys. Fluids 28, 053107 (2016).

[52] A. Zöttl and H. Stark, Emergent behavior in active colloids, J. Phys.: Condens. Matter 28, 253001 (2016). 